\title{
Le kyste osseux anévrysmal de la mandibule : à propos de deux cas
}

\section{Aneurysmal bone cyst of the mandible: two cases report}

\author{
ABDELFATEH SLAMA ${ }^{1}$, NAILA FARHAT ${ }^{1}$, MONTASSER BOUZAIĖNE ${ }^{1}$, TAHAR YACOUBI ${ }^{2}$, HABIB KHOCHTALI ${ }^{1}$
}

\section{RÉSUMÉ}

Le kyste osseux anévrysmal des maxillaires est exceptionnel et sa symptomatologie n'est pas spécifique. Les auteurs rapportent 2 cas de kyste anévrysmal de la mandibule.

La première observation concerne une jeune fille, âgée de 6 ans, qui a consulté pour une asymétrie faciale en rapport avec une masse latéro-mandibulaire droite ; l'aspect radiologique était celui d'une image radiotransparente multiloculaire. La deuxième observation concerne un garçon, âgé de 11 ans, qui présentait une tuméfaction osseuse pré-auriculaire gauche; l'examen radiologique montrait une image kystique uniloculaire dans le condyle. L'examen anatomopathologique de la pièce de curetage a conclu au diagnostic de kyste anévrysmal dans les 2 cas. L'évolution a été marquée par une récidive dans le premier cas, qui a nécessité une reprise chirurgicale. Med Buccale Chir Buccale 2007; 13 : 139-142.

médecine buccale chirurgie buccale

VOL. $13, \mathrm{~N}^{\circ} 3$ 2007

page 139

mots clés: mandibule, kyste anévrysmal

\section{SUMMARY}

The aneurysmal bone cyst is a benign bone dystrophy which occurs exceptionally the jaws. The clinical and radiological patterns are not specific. The diagnosis is confirmed by the histological examination. The authors report two cases of aneurysmal mandibular cyst.

The first patient was a 6-year-old girl who complained for a facial asymmetry secondary to a right mandibular mass. CT-scan revealed a large multilocular radiolucent lesion.

The second patient was an 11 year-old boy who consulted for a left pre-auricular swelling. The radiographic examination showed a unilocular cyst lesion of the condyle. The histological examination concluded to the diagnosis of aneurismal mandibular cyst in both cases. After surgical treatment, a recurrence occurred in the first case and needed operation. Med Buccale Chir Buccale 2007; 13 : 139-142.

key words: mandible, aneurysmal cyst 
Le kyste osseux anévrysmal est une dystrophie osseuse bénigne qui forme une lésion cavitaire uni- ou multiloculaire, à contenu hématique, non bordée par un endothélium. II a été décrit, en 1942, par Jaffe et Lichtenstein comme une entité pathologique distincte ${ }^{[1]}$. II siége souvent dans la métaphyse des os longs et le rachis ${ }^{[1,2]}$. Le kyste anévrysmal des maxillaires est exceptionnel et représente 1,5\% des kystes non odontogéniques [3]. Les auteurs rapportent deux cas de kyste osseux anévrysmal de la mandibule en insistant sur les difficultés diagnostiques de cette entité rare.

\section{PREMIÈRE OBSERVATION}

En 1999, l'enfant R.B, âgée de 6 ans, s'est présentée pour une tuméfaction mandibulaire droite

médecine

buccale

chirurgie

buccale

VOL. $13, \mathrm{~N}^{\circ} 3$ 2007

page 140 évoluant depuis 3 mois (Fig.1). L'examen exobuccal montrait une asymétrie faciale en rapport avec une tuméfaction osseuse, indolore, occupant l'hémimandibule droite, sans troubles sensitifs. A l'examen endobuccal, on notait un léger trismus avec

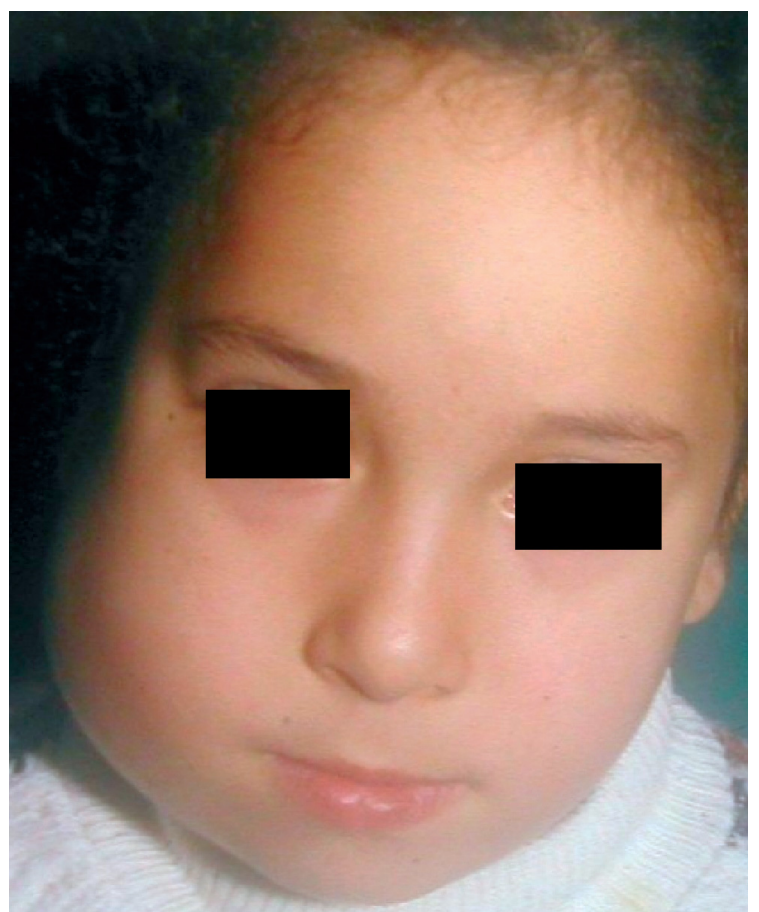

Figure 1 : Tuméfaction latéro-mandibulaire droite responsable d'une asymétrie faciale.

Facial asymmetry secondary to a right mandibular swelling. une soufflure des corticales. La radiographie panoramique objectivait une image radioclaire, cloisonnée, bien limitée occupant l'angle et la branche montante mandibulaire droite (Fig. 2). L'examen tomodensitométrique montrait une image multiloculaire avec rupture de la corticale interne. Le diagnostic d'améloblastome et de myxome a été évoqué, sans éliminer un kyste anévrysmal. La patiente a été opérée par voie d'abord externe, sous angulo-mandibulaire, et il a été réalisé un curetage de la lésion kystique. L'examen anatomopathologique a confirmé le diagnostic de kyste anévrysmal. Au $10^{\circledR}$ mois postopératoire, on a observé une récidive kystique nécessitant une reprise chirurgicale avec exérèse large de la lésion. Après un suivi de 5 ans, il n'a pas été noté de récidive et on pouvait observer à une normalisation de la morphologie faciale.

\section{DEUXIÈME OBSERVATION}

En 2004, l'enfant S.R, âgé de 11 ans, ayant des antécédents de traumatisme mandibulaire, a consulté pour une tuméfaction pré-auriculaire gauche évoluant depuis 8 mois. L'examen facial a objectivé la présence d'une tuméfaction osseuse indolore, mesurant $3 \mathrm{~cm}$ dans son grand axe, mobile avec les mouvements du condyle mandibulaire gauche, n'entrainant pas de limitation de l'ouverture buccale. L'examen endobuccal montrait une voussure mandibulaire intéressant la corticale interne de la branche montante, recouverte par une muqueuse d'aspect normal. II n'y avait pas de

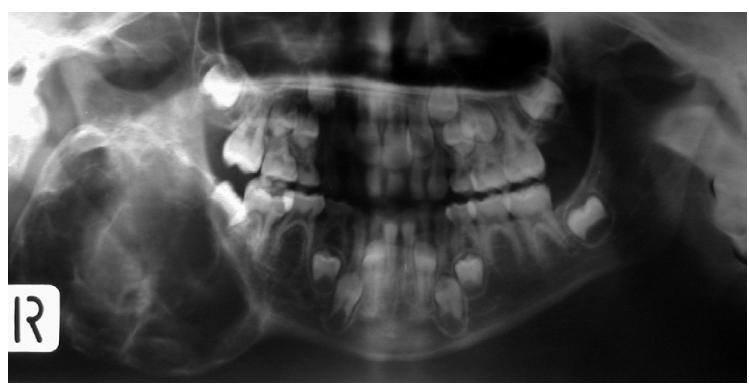

Figure 2 : Radiographie panoramique montrant une image radiotransparente et multiloculaire de la mandibule droite.

Panoramic radiograph showing a multilocular radiolucent lesion of the right mandible. 
troubles de l'articulé dentaire. L'orthopantomogramme a révélé la présence d'une image ostéolytique bien limitée du ramus et du condyle mandibulaire gauches. L'examen tomodensitométrique montrait une image kystique, uniloculaire avec soufflure des corticales (Fig. 3).

Le patient a été opéré par voie endobuccale et un curetage appuyé de la cavité kystique a été réalisé. L'examen anatomopathologique a conclu au diagnostic de kyste anévrysmal du ramus et du condyle. Le prélèvement était constitué de tissu osseux périphérique de type compact et d'une lésion kystique comportant des cloisons fibreuses renfermant des fibroblastes, des sidérophages, des structures capillaires, des travées ostéoides et quelques ostéoclastes. La cavité qui avait un contenu hématique, était revêtue par un pseudoendothélium (Fig. 4). L'évolution postopératoire a été favorable, sans récidive après 24 mois.

\section{COMMENTAIRE}

Le kyste osseux anévrysmal représente 1 à $4 \%$ de toutes les tumeurs et dystrophies osseuses bénignes [3,4]. II peut se développer au niveau de tous les os du squelette avec une prédilection pour les os longs et le rachis. La localisation mandibulaire, rare, touche par ordre décroissant le corps (40\%), le ramus (30\%), l'angle (19\%), la

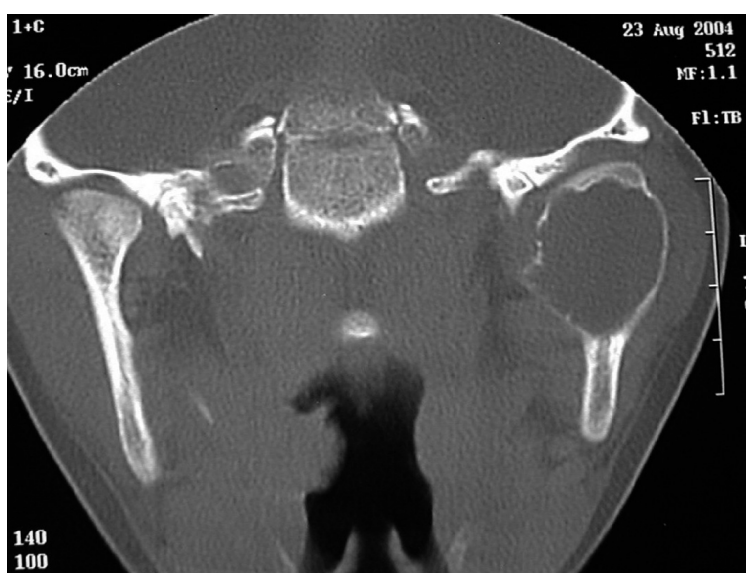

Figure 3 : Examen tomodensitométrique (coupe coronale) montrant une image kystique, uniloculaire avec soufflure des corticales.

CT scan (coronal plane) revealing an unilocular cyst lesion with cortical expansion. symphyse (9\%), et le condyle (2\%) [3,5]. Le kyste anévrysmal de la mandibule survient généralement avant l'âge de 20 ans ${ }^{[1,5]}$. II semble exister une prédominance féminine, non admise par tous les auteurs [3,6].

L'étiopathogénie du kyste osseux anévrysmal reste encore incertaine. La transformation d'une lésion primitive a été suggérée par Ewing et serait liée à des phénomènes hémorragiques [7]. Le kyste osseux anévrysmal était considéré par Lichtenstein comme une lésion de nature vasculaire due à une énorme dilatation capillaire en rapport avec une élévation de la pression veineuse ${ }^{[1]}$. Sanerkin a décrit en 1983, à coté de la forme vasculaire habituelle, une variante solide du kyste osseux anévrysmal caractérisée par l'absence de cavités à contenu hématique ${ }^{[8]}$. L'étiologie posttraumatique a été évoquée par certains auteurs ${ }^{[4,7]}$; la notion de traumatisme a été retrouvée dans la $2^{\mathrm{e}}$ observation.

La symptomatologie clinique n'est pas spécifique. C'est souvent une tuméfaction osseuse indolore, d'évolution lente et responsable d'une asymétrie faciale. Des déplacements dentaires, avec conservation de la vitalité, sont parfois observés [3,7]. Radiologiquement, le kyste anévrysmal de la mandibule se traduit, habituellement, par une image ostéolytique multiloculaire, bordée par un fin liséré

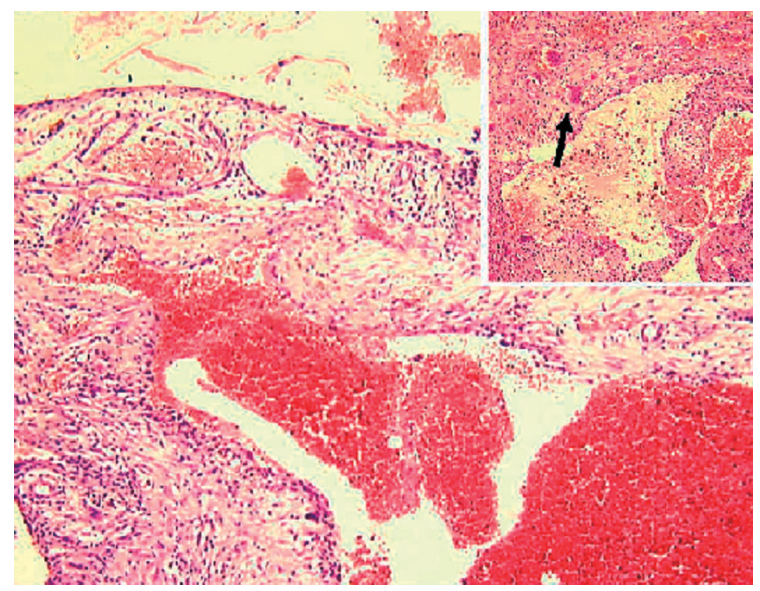

Figure 4 : Cavité anévrysmale pseudo-vasculaire, remplie de sang, entourée par une membrane fibreuse. En haut et à droite : il existe de nombreux ostéoclastes autour des petites cavités (HE, x50).

Pseudovascular aneurysmal cavity filled by blood and surround by fibrous membrane. Upper right: presence of numerous osteoclasts surrounding small cavities $(H E$, $x 50)$. médecine

buccale

chirurgie

buccale

VOL. $13, N^{\circ} 3$ 2007

page 141 
d'ostéocondensation et présentant parfois des cloisons osseuses. A coté de cet aspect radiologique habituel (image radioclaire) des images mixtes, voire radio-opaques ont été décrites ${ }^{[1,5]}$. L'examen tomodensitométrique permet d'apprécier l'étendue de la lyse osseuse et de montrer la soufflure des corticales pour les lésions évoluées ${ }^{[3]}$. Cet aspect a été observé dans la $2^{\mathrm{e}}$ observation. Devant ces aspects cliniques et radiologiques peu caractéristiques, le diagnostic différentiel fait évoquer un myxome, un kyste solitaire, un hémangiome ou un granulome central à cellules géantes ${ }^{[3,5]}$. Le diagnostic définitif est

\section{RÉFÉRENCES}

médecine buccale chirurgie buccale

VOL. $13, \mathrm{~N}^{\circ} 3$ 2007

page 142
1 - Reyneke JP. Aneurysmal bone cyst of the maxilla. Oral Surg 1978 ; 45 : 441-6.

2 - Tuna H, Karatas A, Yilmaz ER, Yagmurlu B, Erekul S. Aneurysmal bone cyst of the temporal bone: case report. Surg Neurol 2003 ; 60 : 571-4.

3 - Perrotti V, Rubini C, Fioroni M, Piattelli A. Solid aneurysmal bone cyst of the mandible. Int $J$ Pediatr Otorhinolaryngol 2004 ; 68 : 1339-44.

4 - Sokolo R, Alseriani F, Lagrange AC, Dumollard JM, Peoc'h M, Seguin P. Kyste osseux anévrysmal du condyle mandibulaire. Rev Stomatol Chir Maxillofac 2006; 107 : 109-14.

5 - Kiottavorncharoen S, Joos U, Brinkschmidt C, Werkmeister R. Aneurysmal bone cyst of the mandible: a case report. Int J Oral Maxillofac Surg 2003 ; 32 : 41922. apporté par l'examen anatomopathologique de la pièce opératoire ${ }^{[7]}$.

Le traitement du kyste osseux anévrysmal de la mandibule est chirurgical, soit conservateur, soit radical $[4,5]$. Le traitement conservateur consiste à réaliser un curetage appuyé de la cavité kystique. Ce curetage risque d'être incomplet dans les lésions étendues et expose à un risque non négligeable de récidive, comme dans la $1^{\mathrm{re}}$ observation. Pour éviter les récidives, certains auteurs préconisent une chirurgie radicale d'emblée avec résection de la lésion et reconstruction immédiate ${ }^{[3,4]}$.
6 - Ginisty D, Mettoudi JD, Adamsbaum C, Dhellemmes C, Maillet S. Tumeurs bénignes et pseudotumeurs des maxillaires de l'enfant. Rev Stomatol Chir Maxillofac 1996 ; $97: 12-6$.

7 - Motamedi MHK. Destructive aneurysmal bone cyst of the mandibular condyle: report of a case and review of the literature. J Oral Maxillofac Surg 2002 ; 60 : 135761.

8 - Sanerkin NG, Mott MG, Roylance J. An unusual intraosseous lesion with osteoblastic aneurysmal and fibromyxoid elements: solid variant of aneurysmal bone cyst. Cancer 1983 ; $51: 2278-82$. 